\title{
Missing malaria? Potential obstacles to diagnosis and hypnozoite eradication
}

\section{Poor specimen collection and limited availability of primaquine may be putting patients at risk}

ecently, one of us experienced an episode of probable malaria on returning from fieldwork in the Solomon Islands. Although a clinical diagnosis of malaria was made, and the illness responded to empirical therapy with artemetherlumefantrine (Riamet, Novartis), a laboratory diagnosis was not achieved.

Suspected malaria in travellers who have returned to Australia from overseas will present without notice and, owing to the often severe nature of this illness, will require immediate attention. This may occur in localities where personal consultation with an infectious diseases physician is not possible. Primaquine for the eradication of malarial hypnozoites from the liver may not be readily available. In this article, we aim to provide brief expert guidance on the diagnosis of malaria, the use of primaquine for eradication therapy and the implications of the limited availability of this treatment in Australia.

Patients presenting with fever should be questioned about their travel history. Clinicians should be mindful that malarial relapse (Plasmodium vivax and P. ovale) or recrudescence (P. malariae) may occur months, or even years, after primary infection. Further, relapse may be the first symptomatic presentation. ${ }^{1}$ Therefore, any patient with pyrexia and a history of travel to an endemic area in the past 3 years might be considered as potentially having malaria. ${ }^{2}$

\section{Initial investigation}

Clinical suspicion should be raised in patients who demonstrate specific symptoms associated with the disease, such as relapsing fever, rigors or chills. Note that immune-naive people may not always present with the typical cyclical fevers of malaria. ${ }^{2,3}$ Unless a separate, simultaneous, pathological process is present (such as concurrent dengue fever, other infections or a non-infectious cause), the presence of localised symptoms, a rash, or the onset of symptoms within the prepatent period (7 days) after initial travel to an endemic area may assist in excluding a diagnosis of malaria. ${ }^{3}$

Laboratory investigation of patients who potentially have malaria requires blood collected in EDTA anticoagulant tubes immediately on presentation. Both thick and thin film microscopy should be requested. As morphological changes in parasites will develop within hours, blood should be delivered to the laboratory within an hour of collection. Immunodiffusion-based rapid diagnostic antigen tests should also be performed if available, but these tests do not supplant microscopy. ${ }^{4}$ Currently, there is no consensus regarding the correct timing and number of specimens required to exclude a diagnosis of malaria. It appears that a single collection is often sufficient for diagnosis. ${ }^{3}$ However, further specimen collections taken shortly after the onset of febrile paroxysms may be necessary for the detection of $P$. falciparum malaria, as this species is sequestered in the deeper microvasculature at other times during its life cycle., ${ }^{2,3}$

Returned travellers who have used malaria prophylaxis may occasionally still acquire malaria, even when they strictly adhere to the dosage regimen. ${ }^{1}$ In such cases, the parasitaemia is often very low, requiring multiple blood collections for diagnosis, but individuals with little or no prior exposure will still be significantly unwell. Very rarely, malaria may be acquired during short stays in endemic areas; for example, during airport stopovers. ${ }^{5}$

\section{The role of PCR}

Polymerase chain reaction (PCR) testing represents a more recent and highly efficacious method for the detection and speciation of malaria in febrile patients. Nevertheless, specimen collection during an afebrile period may lead to a false-negative PCR test result. Due to its expense and limited availability, PCR testing is currently restricted to confirmation and speciation, or cases where a malaria diagnosis is strongly suspected but microscopy and antigen testing are negative.

\section{Primaquine}

Infection with relapsing species of malaria ( $P$. vivax and $P$. ovale) requires eradication of hypnozoites from the patient's liver using primaquine. P. ovale malaria requires half the dose of primaquine used in cases of $P$. vivax. Some strains of $P$. vivax acquired in the South Pacific and South-East Asia may need higher doses of primaquine for eradication. ${ }^{6}$ Tests 
for glucose-6-phosphate dehydrogenase deficiency should be performed on all patients before primaquine therapy, in order to avoid potentially lifethreatening oxidative events in enzyme-deficient individuals. Currently, primaquine is erratically available in hospital pharmacies and may not be stocked at all in smaller, regional facilities. Also, it cannot be accessed under the Pharmaceutical Benefits Scheme, despite being indicated in Australian therapeutic guidelines. ${ }^{6}$ These factors limit its availability to hospitals and community pharmacies. For example, when malaria presents and is treated in general practice, the limited availability of primaquine could result in this important therapy not being administered, especially in regional, rural and isolated areas.

In summary, given increasing rates of travel to endemic areas by Australians, clinicians may be faced with a case of malaria at any time.

Hence, it is important that they have the correct specimen-collection and treatment protocols at hand. Primaquine should be available through the Pharmaceutical Benefits Scheme to patients treated in a community setting.

Competing interests: No relevant disclosures.

Provenance: Not commissioned; externally peer reviewed.

1 Morgan GS, Chiodini P, Evans M. Relapsing malaria: two cases of malaria presenting 8 months after return from Africa despite adherence to antimalarial chemoprophylaxis. Br J Gen Pract 2012; 62: 555-556.

2 Rogers WO. Plasmodium and Babesia. In: Versalovic J, Carroll KC, Funke G, et al, editors. Manual of clinical microbiology. 10th ed. Washington, DC: ASM Press, 2011: 2091-2112.

3 Fairhurst RM, Wellems TE. Plasmodium species (malaria). In: Mandell GL, Bennett JE, Dolin R, editors. Mandell, Douglas, and Bennett's principles and practice of infectious diseases. 7th ed. Philadelphia: Churchill Livingston Elsevier, 2010: 3437-3462.

4 Wilson ML. Laboratory diagnosis of malaria: conventional and rapid diagnostic methods. Arch Pathol Lab Med 2013; 137: 805-811.

5 Cimerman S, Barata LC, Pignatari AC, et al. Malaria transmission associated with airplane travel. Braz J Infect Dis 1997; 1: 135-137.

6 Antibiotic Expert Group. Therapeutic guidelines: antibiotic. Version 14. Melbourne: Therapeutic Guidelines Limited, 2010: 166. 